Salamanca-López, D., Suárez-Rico, Y. \& GimenoRuiz, M. (2021). Explorando la divulgación de información integrada por universidades colombianas. Un análisis de páginas web basado en el marco del IIRC. Contaduría Universidad de Antioquia, 79, 161-192. Doi: https://doi. org/10.17533/udea.rc.n79a07

\title{
Explorando la divulgación de información integrada por universidades colombianas. Un análisis de páginas web basado en el marco del IIRC
}

Diana Yamile Salamanca-López

Universidad Nacional de Colombia dysalamancal@unal.edu.co

Orcid: 0000-0002-9937-3987

Yuli Marcela Suárez-Rico Universidad Nacional de Colombia ymsuarezr@unal.edu.co Orcid: 0000-0002-3754-6600

María Amparo Gimeno-Ruiz

Universidad de Valencia amparo.gimeno@uv.es

Orcid: 0000-0003-4555-1810 
Explorando la divulgación de información integrada por universidades colombianas. Un análisis de páginas web basado en el marco del IIRC

Resumen: La información integrada se presenta como un fenómeno de reciente auge que permite a diversas organizaciones informar de una manera simplificada pero completa sobre su gestión respecto a diversos tipos de capitales. Aunque predominantemente se ha desarrollado en las empresas privadas, en el ámbito internacional viene reconociéndose que otro tipo de organizaciones como las universidades, divulgan esta información o cuentan con la necesidad y el potencial para ello. Este trabajo tiene como objetivo analizar en qué medida la información solicitada para construir un reporte integrado de acuerdo con los requerimientos del Marco Conceptual del IIRC, ya está revelada en internet por las universidades colombianas incluidas en el ranking QS 2019. Para ello, se construyó un índice de cumplimiento a partir de una lista de verificación. Los resultados sugieren que el nivel de divulgación es heterogéneo entre universidades, siendo el modelo de negocio la unidad de análisis más divulgada.

Palabras Clave: Información Integrada; Universidades; Ranking QS 2019; Colombia; IIRC

Exploring disclosure of integrated information by Colombian universities. A web page analysis based on the IIRC framework

Abstract: Integrated reporting is a recently emerging phenomenon allowing various organizations to report on their management of different types of capital in a simplified but comprehensive manner. Although Integrated reporting has mainly been developed in private companies, at global level it has been recognized that other types of organizations, such as universities, disclose this information or have the need and potential to do so. This paper aims to analyze to what extent the information requested to build an integrated report, in accordance with the requirements of the IIRC Conceptual Framework, is already disclosed on the internet by the Colombian universities included in the QS 2019 ranking. To this end, a compliance index was constructed based on a checklist. The results suggest that the level of disclosure is heterogeneous across universities, with the business model being the most disclosed unit of analysis.

Keywords: Integrated Information; Universities; QS 2019 ranking; Colombia; IIRC

Explorando a divulgação de informação integrada por universidades colombianas. Uma análise de páginas web baseada no marco do IIRC

Resumo: A informação integrada se apresenta como um fenômeno de recente auge que permite a diversas organizações informar de uma maneira simplificada, mas completa sobre sua gestão respeito de diversos tipos de capitais. Embora predominantemente tenha se desenvolvido nas empresas privadas, no ambito internacional vem se reconhecendo que outro tipo de organizações como as universidades, divulgam esta informação ou contam com a necessidade e o potencial para isso. Este trabalho tem como objetivo analisar em que medida a informação solicitada para construir um relatório integrado de acordo com os requerimentos do Marco Conceptual do IIRC, já está revelada na internet pelas universidades colombianas incluídas no ranking QS 2019. Para isso, construiu-se um índice de cumprimento a partir de uma lista de verificação. Os resultados mostram que o nível de divulgação é heterogêneo entre universidades, sendo o modelo de negócio a unidade de análise mais divulgada.

Palavras chave: Informação integrada; Universidades; Ranking QS 2019; Colômbia; IIRC

L'Exploration de la divulgation intégrée de l'information par les universités colombiennes. Une analyse des pages web basée sur le cadre IIRC

Résumé: Le rapport intégré est un phénomène récemment apparu qui permet à diverses organisations de rendre compte, de manière simplifiée mais complète, de leur gestion de divers types de capitaux. Bien qu'elle ait été développée principalement dans les entreprises privées, il a été reconnu au niveau international que d'autres types d'organisations, comme les universités, divulguent ces informations ou ont le besoin et le potentiel de le faire. Cet article vise à analyser dans quelle mesure les informations requises pour élaborer un rapport intégré conformément aux exigences du cadre conceptuel de l'International Integrated Reporting Council (IIRC) sont déjà divulguées sur Internet par les universités colombiennes figurant dans le classement QS 2019. À cette fin, un indice de conformité a été construit sur la base d'une liste de contrôle. Les résultats suggèrent que le niveau de divulgation est hétérogène entre les universités, le modèle d'entreprise étant l'unité d'analyse la plus divulguée.

Mots clés: rapport intégré, universités, classement QS2019, Colombie, IIRC 
Cont. udea (julio-septiembre, pp. 161-192. (c) Universidad de Antioquia-2021.

\title{
Explorando la divulgación de información integrada por universidades colombianas. Un análisis de páginas web basado en el marco del IIRC ${ }^{1}$
}

\author{
Diana Yamile Salamanca-López, Yuli Marcela Suárez-Rico y María Amparo Gimeno-Ruiz \\ https://doi.org/10.17533/udea.rc.n79a07
}

Primera versión recibida en abril de 2021 - Versión aceptada en julio de 2021

\section{Introducción}

Debido a fenómenos como el calentamiento global, los desequilibrios sociales y las cada vez más frecuentes crisis económicas y sociales, en las últimas décadas han surgido de manera aislada y con enfoques diferentes, innumerables iniciativas para informar de la influencia de dichos aspectos en las entidades tanto públicas como privadas, así como las acciones que afectan el entorno en el que operan. Podemos destacar como ejemplos, los estándares internacionales de gobierno corporativo, las iniciativas de Responsabilidad Social Corporativa, los estándares GRI o las Normas ISO, cuyo enfoque no siempre es convergente, lo que repercute en la producción de varios informes, muchas veces desconectados entre sí.

A partir de esta situación, se plantea la necesidad de una gestión diferente de las organizaciones y de la forma de comunicar sus resultados. En este contexto surge el reporte integrado -IR-, como una innovación que promueve el pensamiento integrado en la gestión para enfocarse en la creación de valor, teniendo como uno de sus objetivos un único informe en el cual se disponga de toda la información de la organización, preparado desde una perspectiva de sostenibilidad (Gómez-Villegas, 2017; IIRC, 2013; Rivera-Arrubla et al., 2016).

Dado que los stakeholders están tomando decisiones sobre la base de información financiera y no financiera combinada, este tema tiene una demanda cada vez mayor (Navarrete Oyarce y Gallizo Larraz, 2019). Prueba de ello son los más de 3700 artículos recogidos en la base de datos de Scopus desde el

1 Las autoras agradecen el aporte financiero recibido del ICETEX. El articulo aporta al foco-reto país Sociedad, desarrollado en el marco del programa Colombia Científica- Pasaporte a la ciencia. 
Explorando la divulgación de información integrada por universidades colombianas...

año 2013, cuando se publicó el Marco Conceptual Internacional del Reporte Integrado (Scopus, 2021). Adicionalmente, el International Integrated Reporting Council -IIRC- ha señalado la importancia de explorar la manera en la que la información integrada es presentada en internet, en tanto con ella se toman las ventajas de las tecnologías emergentes para lograr interconexiones y entregar un mejor sentido a los stakeholders (IIRC, 2012; Lodhia y Stone, 2017). El uso de internet como un mecanismo de difusión de información integrada podría favorecer el principio de conectividad que implica la posibilidad de tender puentes entre horizontes de desempeño, efectuar conexiones efectivas entre distintos tipos de información y conectar usuarios con la empresa para responder de mejor forma a sus intereses y expectativas (IIRC, 2021; Rivera-Arrubla y ZorioGrima, 2016).

Muchos de los estudios realizados hasta ahora se han enfocado en los reportes de empresas en Sudáfrica y en el Reino Unido. El primero por ser el único país donde es obligatoria su presentación para empresas que cotizan en la bolsa (Solomon y Maroun, 2012) y el segundo por ser un país que tiene tradición de realizar reportes financieros (Akhter y Ishihara, 2018).

Recientemente, varios trabajos han explorado la divulgación de información integrada en otro tipo de organizaciones y entre ellas destacan las universidades, que por su naturaleza y características son vistas de una parte, como un motor de cambio social y de otra, como organismos independentes que también están inmersos en dinámicas propias de la globalización como son la competencia y la necesidad de generar confianza a través de la transparencia en su gestión (Brusca et al., 2018; Guthrie et al., 2020; Iacuzzi et al., 2020; Veltri y Silvestri, 2015).

Si bien una de las cuestiones centrales del IIRC recae en promover un único informe que integre la rendición de cuentas de diversos capitales, un primer paso podría estar en el diagnóstico de qué contenidos dispuestos en el marco ya se encuentran divulgados por las organizaciones a través de sus otros informes. Puntualmente, en el ámbito de las universidades esta exploración permitiría hacer un rastreo de qué tanta información relacionada con los contenidos que el marco reclama, ya se encuentra disponible por estas instituciones. Esto, teniendo en cuenta que, aunque el informe integrado se presenta como una innovación en el reporting, a la fecha no ha alcanzado una aplicación generalizada en el ámbito de las universidades colombianas y tampoco existe evidencia de que las organizaciones que declaran aplicar el marco del IIRC ya hayan efectuado cambios al nivel estratégico y de sus prácticas corporativas a nivel internacional (Humphrey et al., 2017).

Así, este estudio atiende el llamado de Bernardi (2020) en el sentido de diagnosticar el camino de diversos tipos de organizaciones en la construcción de informes integrados, considerando además que en el caso de las universidades 
colombianas, podría constituirse en un primer acercamiento a los contenidos esperados para este tipo de reporte.

A la fecha son pocas las investigaciones que se han realizado sobre información integrada en Colombia, centrándose las existentes en los reportes integrados de las empresas. Ejemplo de ello lo constituyen los trabajos de Correa-García, et al.(2016), Macías y Farfán-Liévano (2017) y Giraldo-López et al. (2018).

Teniendo en cuenta lo anterior, este artículo tiene como objetivo analizar en qué medida la información solicitada para construir un reporte integrado, de acuerdo con los requerimientos del Marco Conceptual del IIRC, ya está revelada en internet por las universidades colombianas incluidas en el ranking QS 2019.

Para ello, el documento se estructura en 5 partes, además de la presente introducción. La primera parte describe brevemente el marco de información integrada emitido por el IIRC. La segunda parte trata el tema de la información integrada en Instituciones de Educación superior. La tercera parte y cuarta parte exponen la metodología utilizada y los resultados obtenidos, consistente en una medición utilizando un índice de cumplimiento. Finalmente, se exponen las conclusiones alcanzadas.

\section{El Marco del IIRC y la información integrada}

El IR surge como una propuesta innovadora del IIRC para desarrollar el pensamiento integrado en la gestión de las organizaciones y en la creación de valor. Así, cada organización centra sus esfuerzos en la consecución de determinados objetivos, buscando una visión global sobre su valor económico o su capacidad para crear valor futuro (Gómez-Villegas, 2017), a la par que supera la elaboración de informes fragmentados y dispersos.

Es importante señalar que la información integrada no se limita a la presentación de informes, sino que, de acuerdo con el IIRC, se trata de proponer un cambio en los procesos internos y en las estructuras de las organizaciones (Rivera-Arrubla et al., 2016). Esto tendrá como resultado la asignación eficiente y productiva de recursos a la vez que beneficia a los grupos de interés, incluyendo los empleados, clientes, proveedores, socios, comunidad local, legisladores, reguladores y políticos (IIRC, 2021).

El informe resultado de dicho proceso, debe ser una "comunicación concisa sobre cómo la estrategia, el gobierno, el desempeño y las perspectivas de una organización, en el contexto de su entorno externo, conducen a la creación de valor a corto, mediano y largo plazo" (IIRC, 2013, p.8).

En 2013, el IIRC publicó el Marco Conceptual Internacional de Información Integrada, consistente en una guía basada en principios que permite flexibilidad y por lo tanto aplicabilidad en países con diversas tradiciones contables y condiciones institucionales. Este marco establece los principios y contenidos que rigen un reporte integrado en términos generales. Si bien este tipo de información ha sido 
Explorando la divulgación de información integrada por universidades colombianas...

pensada para el sector privado y específicamente para compañías con fines de lucro, el Marco Conceptual también expresa que puede adaptarse a otro tipo de organizaciones.

Recientemente, el IIRC emitió una revisión del marco. Las modificaciones del mismo están enfocadas a una simplificación de la declaración de responsabilidad, una distinción entre productos y resultados y un énfasis en la presentación de informes equilibrados (IIRC, 2021).

Un aspecto central de Marco es la creación del valor, entendida como un proceso simbiótico con el entorno externo y que se exterioriza en un "aumento, disminución o transformación de los capitales causados por las actividades y las salidas de la organización" (IIRC,2013, p.11).

En este proceso, el Marco Conceptual hace referencia a los capitales que define como "inventarios de valor que aumentan, disminuyen o se transforman a través de las actividades y salidas de la organización”, (2013, p. 12). En la tabla 1, se muestra la clasificación de los capitales y un resumen de su definición.

Tabla 1. Tipos de capital.

\section{Tipos de capital}

Financiero Fondos de los cuales dispone la organización para su uso en el desarrollo de sus actividades. Pueden ser obtenidos a través de la financiación, capital o subvenciones o generados por la organización.

Industrial Bienes físicos fabricados que están disponibles para el uso en la producción de bienes o servicios de la organización, incluye edificios, equipos e infraestructura.

Intelectual Intangibles que se basan en el conocimiento de la organización, incluyen la propiedad intelectual (patentes, derechos de autor, software, derechos y licencias) y el capital organizacional (conocimiento tácito, sistemas, procedimientos y protocolos).

Humano Competencias, capacidades y experiencias de las personas y sus motivaciones para innovar, incluye aspectos tales como la lealtad, enfoque de gestión de riesgos y valores éticos de una organización.

Social y Instituciones y relaciones dentro y entre las comunidades, los grupos de interés y otras

Relacional redes, así como a la capacidad de compartir información para mejorar el bienestar individual y colectivo

Natural Recursos y procesos medioambientales renovables y no renovables, que incluyen: aire, agua, minerales, bosques, biodiversidad y la salud del ecosistema.

Fuente: Elaboración propia a partir de Marco Conceptual (IIRC, 2021)

El informe integrado debe incluir toda la información relevante para que los diferentes usuarios puedan tomar decisiones. Para ello establece siete directrices que guían el proceso de preparación, el contenido y la presentación de la información (IIRC, 2013). Estas directrices son: i) Enfoque estratégico y orientación futura; ii) Conectividad de la información; iii) Relaciones con los grupos de interés; iv) Materialidad; v) Concisión; vi) Fiabilidad y exhaustividad y vii) Consistencia y comparabilidad. Sin embargo, es importante destacar que el 
Marco no establece indicadores clave de desempeño ni métodos de divulgación de asuntos específicos.

Los contenidos son otro aspecto fundamental. El IIRC los clasifica en ocho categorías, descritos a continuación con la correspondiente pregunta direccionadora:

1. Descripción General de la organización: ¿Qué es lo que hace la organización y cuáles son las circunstancias en las que opera?

2. Gobierno Corporativo: ¿De qué manera la estructura de gobierno corporativo de la organización apoya la capacidad de crear valor en el corto, medio y largo plazo?

3. Modelo de Negocio: ¿Cuál es el modelo de negocio de la organización?

4. Riesgos y Oportunidades: ¿Cuáles son los riesgos y las oportunidades específicas que afectan la capacidad de la organización para crear valor en el corto mediano y largo plazo y como les está haciendo frente la organización?

5. Estrategia y asignación de recursos: ¿De dónde viene la organización, hacia dónde quiere ir, y cómo pretende llegar?

6. Desempeño: ¿En qué medida la organización alcanza sus objetivos estratégicos y cuáles son los resultados en términos que tengan efecto sobre los capitales?

7. Perspectivas: ¿A qué retos e incertidumbres puede enfrentarse la organización al desarrollar su estrategia?, y ¿Cuáles son las implicaciones potenciales para su modelo de negocio y desempeño futuro?

8. Bases para la elaboración y presentación al hacerlo: ¿A qué retos e incertidumbres puede enfrentarse la organización al desarrollar su estrategia?, y ¿Cuáles son las implicaciones potenciales para su modelo de negocio y desempeño futuro?

9. Orientación general del informe: ¿Cómo determina la organización los asuntos que deberán ser incluidos en el IR y como esos asuntos son cualificados o evaluados?

\section{II.1 Ventajas y críticas a la información integrada}

Aunque no existe una forma de publicación del IR única e infalible para todas las organizaciones, diversos estudios señalan que las ventajas de la implementación de los IR son muchas (Rowbottom y Locke, 2015). Una primera ventaja de los IR, encontrada en la literatura está dada en el aspecto financiero por menores costos de capital, mayor liquidez y mayores flujos de efectivo esperados. En esta línea se encuentran los trabajos de Churet y Eccles (2014); Tascón et al. (2015); Lee y Yeo (2016); De Villiers et al. (2016), y Navarrete-Oyarce y Gallizo-Larraz (2019).

Otra ventaja recurrente en la literatura es la relacionada con la mejora en la percepción de la organización por los stakeholders. En ese sentido, Adhikariparajuli et al., (2019)reflecting integrated thinking, are disclosed voluntarily 
Explorando la divulgación de información integrada por universidades colombianas...

and whether HEI specific features influence the resulting disclosures. Design/ methodology/approach-Existing IR guidelines given by the International Integrated Reporting Council (IIRC indican que, cuando las organizaciones adoptan un nuevo estándar o normativa tienen más probabilidades de obtener aprobación externa, lo que está de acuerdo con la teoría institucionalista. Al extrapolarlo al $I R$, las organizaciones pioneras en la adopción de IR se convertirán en modelos de organización a seguir.

Una tercera ventaja destacada en la literatura es la disminución en el número de reportes que las organizaciones deben emitir. Al respecto, Havlova (2015) citado por Navarrete Oyarce y Gallizo Larraz (2019) estudió 48 empresas del programa piloto cinco años después de la implementación de IR, encontrando que las empresas habían cumplido el objetivo de la emisión de un reporte único. En el contexto de las empresas sudafricanas se han realizado varias investigaciones con resultados similares, entre ellas las realizadas por Solomon y Maroun (2012), quienes estudiaron el impacto de los IR en 10 empresas encontrando que, despues de su implementación hubo mayor revelación e integración de la información social, ambiental y ética y una mayor importancia al riesgo y al manejo del mismo.

Otra de las ventajas asociadas a la implementación, es que el modelo IR, aumenta la toma de decisiones basada en estos informes (Gómez-Villegas, 2017; Richardson y Kachler, 2017), ya que la multiplicidad de informes hace dificil tomar decisiones con información segmentada, mientras que con el IR en un solo informe se puede tener la visión holística de la organización.

Finalmente, algunos trabajos destacan el aumento de la transparencia a nivel interno y externo de las organizaciones (Adams y Simnett, 2011; Navarrete Oyarce y Gallizo Larraz, 2019), la posibilidad de comparar de diferentes empresas (AECA, 2011) lo cual es vital en un mundo de competencia, y la mejora en la información en aspectos sociales, medioambientales, e información sobre ética (Solomon y Maroun, 2012).

A pesar de las ventajas señaladas, también se encuentran posturas críticas respecto a este tipo de informes. Una de las más recurrentes se enfoca en el IIRC y su composición, dada la gran influencia, a través del lobbying, que tuvieron las grandes empresas prestadoras de servicios en la elaboración del Marco Conceptual (Reuter y Messner, 2015; Rivera-Arrubla et al., 2016) y que se refleja en el enfoque del Marco dirigido hacia los intereses de los inversionistas.

Con esto, Dumay et al. (2016) señalan que el Marco Conceptual pasó de un enfoque de sostenibilidad a uno netamente inversionista cuyo propósito principal es "explicar a los proveedores de capital financiero como la organización crea valor a lo largo del tiempo" (IIRC, 2013, p. 8). Como consecuencia de ello, existe la preocupación de que el IR se convierta de manera preponderante en 
un nuevo mercado para las empresas de consultoría y para los contadores en los procesos de implementación y posterior aseguramiento (Gómez-Villegas, 2017).

Por otra parte, también se critica el hecho de que el mismo IIRC no tenga una composición representativa de todos los sectores, lo que se traduce en una ausencia de discusión sobre el IR con puntos de vista diferentes (Dumay et al., 2016).

En cuanto a su aplicación para el sector público existen dudas de la capacidad del IR de integrar la información presupuestaria, financiera y no financiera (Gómez-Villegas, 2017). Aunque el IIRC dispone de una red específica para el sector público y ha publicado documentos relacionados, como el «Integrated thinking and reporting. Focusing on value creation in the public sector», aún no se han realizado adaptaciones al Marco Conceptual. Al respecto, Gómez Villegas (2017) se atreve a proponer la inclusión de otras categorías para los capitales, por ejemplo, el político y el público, para obtener resultados en justicia, equidad y legitimidad.

Desde el punto de vista pragmático, la principal crítica que se evidencia es la ambiguiedad en la manera como debería estructurarse un IR. No está claro qué información debe incluirse, dado que -aunque se tienen principios-, no hay un contenido prescriptivo que caracterice un IR de alta calidad (de Villiers et al., 2014; Dumay et al., 2016; Navarrete Oyarce y Gallizo Larraz, 2019; Solomon y Maroun, 2012), por lo cual el IR puede convertirse en un documento de simple cumplimiento.

También se encuentran críticas a la materialidad, ya que es un concepto complejo que no se ha discutido lo suficiente ni se han llegado a las conclusiones necesarias para poderlo aplicar en los IR (Solomon y Maroun, 2012).

Finalmente, otra crítica es la relacionada con los costos indirectos asociados a la revelación de información, en concreto a la divulgación de información beneficiosa para la competencia, costos políticos y costos legales, los cuales son difíciles de identificar y medir (Correa-García et al., 2016), convirtiéndose por tanto, en una desventaja competitiva. Sobre todo, al revelar el segmento de la operación que más genera beneficios, las innovaciones tecnológicas y los planes de desarrollo.

\section{Las instituciones de educación superior (IES) y el reporte integrado}

Las IES son un sector interesante para estudiar debido a su doble función, de un lado, por ser elementos críticos en una sociedad basada en el conocimiento, debido a su naturaleza de productor y proveedor de conocimiento; por el otro, las universidades al igual que las demás organizaciones son organismos independientes, que tienen en sus órganos rectores a los responsables de la gestión de la institución y de planear su futuro (Low et al., 2015).

El sector de la educación superior ha sido proclive a la revelación de información no financiera, y algunas instituciones incluso publican memorias de 
Explorando la divulgación de información integrada por universidades colombianas...

sostenibilidad con las guías GRI (Brusca y Labrador, 2017). En la base de datos de GRI, a nivel mundial hay 159 universidades que publican memorias bajo esas guías (GRI, 2021), y en Colombia, hay once que publican o han publicado memorias bajo dicho marco.

En los últimos años las IES han tenido cambios en la gestión de su administración, debido a que los ingresos ya no provienen totalmente de los gobiernos (Low et al., 2015) y a que se han incorporado instrumentos de gestión procedentes del ámbito empresarial (Gómez-Villegas, 2017). Esto ha supuesto que se convierta en un sector altamente competitivo por los recursos públicos, por lo que los stakeholders se interesan por el uso efectivo de estos y por el aumento de la transparencia de la información (Veltri y Silvestri, 2015).

También la tecnología y el uso masivo de internet ayudan a la masificación y divulgación de la información, ya que a través de ella se logra llegar a múltiples usuarios, a costos muy reducidos (Navarrete Oyarce y Gallizo Larraz, 2019), en este contexto las universidades también hacen esfuerzos para presentar sus resultados, no solo de aspectos financieros.

En cuanto al número de IES que revelan información sobre sostenibilidad, y más aún en cuanto a la implementación de IR, podría señalarse que el sector se encuentra en una etapa temprana (Adhikariparajuli et al., 2019). En la actualidad, tal y como señalan Brusca y Labrador (2017), ya existen demandas para que las universidades adopten esta práctica, entre ellas, un grupo de directores financieros de las universidades británicas.

Sin embargo, en la base de datos del IIRC de las organizaciones que implementaron el marco o que hacen parte de la red de la organización, solo hay un reporte de universidades y es el perteneciente a The University of Edinburg (IIRC, 2019).

De acuerdo con de Villiers et al. (2016), algunas de estas organizaciones pueden no catalogar sus informes como IR y cumplir con las condiciones para serlo. Asimismo, algunas organizaciones pueden declarar que revelan su información mediante un IR y no cumplir con las condiciones necesarias. A manera de ejemplo, la University of the Free State es una Institución de Educación Superior -IES- que, en el reporte anual de 2018, declaró aplicar el Código King IV, desde 2016 (University of The Free State, 2018). Sobre esta universidad, Veltry y Silvestry (2015) hacen un estudio de caso, encontrando que aunque el informe dice cumplir con el Marco Conceptual, el contenido del reporte no lo ratifica.

En el ámbito colombiano, las IES son entidades que tienen el reconocimiento como prestadoras del servicio público de la educación superior en el territorio colombiano. El Ministerio de Educación Nacional -MEN- las cataloga según su naturaleza jurídica en privadas o públicas, aunque independientemente de esa condición, cada establecimiento educativo debe elaborar y ejecutar un Proyecto Educativo Institucional -PEI-, el cual debe tener entre otros los principios y fines del establecimiento, los recursos disponibles y el sistema de gestión. 
Adicionalmente, la Ley 872 de 2003, reglamentada con el Decreto 1083 de 2015, creó el sistema de gestión de calidad para el sector público; por lo cual las universidades públicas tuvieron que implementar con carácter obligatorio la Norma Técnica de Calidad en la Gestión Pública -NTCGP 1000-(Ley 872, 2003; Decreto 1083, 2015). Esta norma implica que la prestación de los servicios se realice con un enfoque basado en procesos y se haga la toma de decisiones basada en el análisis de datos (Rodríguez, 2019).

Mediante la ley 1712 de 2014, conocida como Ley de Transparencia y del Derecho de Acceso a la Información Pública Nacional, todas "las personas naturales y jurídicas, públicas o privadas, que presten función pública, que presten servicios públicos respecto de la información directamente relacionada con la prestación del servicio público" (Congreso de la República, 2014) deben realizar divulgación de información en la que se incluya la descripción de su estructura orgánica, sus funciones y deberes, la ubicación, divisiones o departamentos, su presupuesto general, las metas y objetivos de las unidades administrativas de conformidad con sus programas operativos y los resultados de las auditorías al ejercicio presupuestal e indicadores de desempeño (Congreso de la República, 2014, articulo 9).

De acuerdo con la información suministrada por el MEN en su página web, existen 52 universidades con calificación de alta calidad, dentro de las cuales 19 son públicas y 33 son privadas.

Varios son los trabajos que han estudiado información integrada en el ámbito de las universidades, como el realizado por Low et al. (2015), que revisa la calidad del capital intelectual revelado por las universidades de Nueva Zelanda, Australia y el Reino Unido, usando un análisis de contenido comparativo. Este estudio encontró que las universidades de los dos primeros paises superaron al Reino Unido en divulgación, siendo Nueva Zelanda la que revela mayor calidad en la información. En España, Gallego- Alvarez et al. (2011) realizaron un estudio similar, analizando el contenido de las páginas de las universidades, con el objetivo de verificar características especiales que podrían influir en el nivel de divulgación, mostrando que a mayor complejidad, internacionalización y rentabilidad, había un mayor grado de divulgación.

Recientemente, Mauro et al. (2020) revisaron los informes de sostenibilidad de las universidades públicas italianas para encontrar qué elementos del IR habían sido incluídos en tales reportes. Sus resultados muestran que hay una alta inclusión de elementos del marco, aunque de una manera poco homogenea y fragmentada.

En cuanto a la implementación de los IR, Adhikariparajuli et al. (2019), analizaron el contenido de 135 reportes de IES del Reino Unido presentados entre el 2014 y 2016, encontrando que hay un incremento en el número de elementos de contenido del IR que reportan las IES. 
Explorando la divulgación de información integrada por universidades colombianas...

En el mismo sentido, el estudio realizado por Oprisor et al. (2017), analizó el nivel de divulgación comparando dos conjuntos de 53 informes de universidades públicas: el primero de ellos formado por IES de primer nivel de todo el mundo y el segundo por IES de Rumania, encontrando que las universidades del primer grupo tienen alto nivel de revelación, mientras que las universidades del segundo grupo tienen un nivel de divulgación significativamente menor.

\section{Diseño metodológico}

\section{IV.1 Muestra}

Para desarrollar nuestro estudio, seleccionamos las universidades colombianas incluidas entre las mejores mil universidades del mundo de acuerdo con el ranking QS del año 2019. La muestra fue basada en criterios de selección, comprensión del problema y pertinencia con el estudio (Galeano, 2004), atendiendo a que este ranking es considerado uno de los más importantes a nivel mundial y se centra no solo en indicadores cuantitativos sobre la producción o el impacto de las investigaciones, sino que también tiene en cuenta aspectos como la ratio de profesores internacionales y la proporción de estudiantes y docentes, entre otros.

Este tipo de selección es válido y utilizado desde un enfoque cualitativo de investigación que busca, de manera selectiva, interpretar los resultados para el grupo de componentes participantes y no pretende extrapolar los hallazgos de manera generalizada para el resto de universidades (Galeano, 2004; Toro Jaramillo y Parra Ramírez, 2010), en tanto se considera muestreo intencional.

Como resultado el estudio se realizó para un total de once universidades, de las cuales cuatro son públicas y siete son privadas, como se aprecia en la tabla 2.

Tabla 2. Universidades colombianas incluidas en el ranking QS World University 2019

\begin{tabular}{llc}
\hline $\begin{array}{c}\text { Posición en } \\
\text { el ranking }\end{array}$ & \multicolumn{1}{c}{ Nombre } & $\begin{array}{c}\text { Según su } \\
\text { Naturaleza }\end{array}$ \\
\hline 272 & Universidad de los Andes & Privada \\
275 & Universidad Nacional de Colombia & Pública \\
407 & Universidad Externado de Colombia & Privada \\
$521-530$ & Pontificia Universidad Javeriana & Privada \\
$701-750$ & Universidad de Antioquia & Pública \\
$801-1000$ & Universidad de La Sabana & Privada \\
$801-1000$ & Universidad del Norte & Privada \\
$801-1000$ & Universidad del Rosario & Privada \\
$801-1000$ & Universidad del Valle & Pública \\
$801-1000$ & Universidad EAFIT & Privada \\
$801-1000$ & Universidad Industrial de Santander - UIS & Pública \\
\hline
\end{tabular}

Fuente: Elaboración propia 
En el proceso de búsqueda y codificación de la información, encontramos que, aunque en años anteriores la Universidad Externado de Colombia, no había publicado informes de gestión, en 2019 publicó un informe de la gestión del rector saliente del periodo 2012 a 2019, el cual fue tomado como fuente de consulta para el presente artículo.

\section{IV.2 Índice de Cumplimiento}

Como herramienta de medición construimos un índice de cumplimiento de los elementos que se incluyen en el Marco Conceptual del IR. De acuerdo con Coy (1995), citado por Guthrie y Abeysekera (2006, p.13) un índice de cumplimento es un "instrumento de investigación que comprende una serie de elementos preseleccionados que, cuando se puntúan, proporcionan una medida que indica un nivel de divulgación en el contexto específico para el que se diseñó el índice”. Este tipo de análisis es el método más utilizado para evaluar la cantidad de información revelada en informes corporativos (Akhter y Ishihara, 2018; Guthrie y Abeysekera, 2006)seeks to examine several research method issues relating to the use of content analysis are examined. Design/methodology/ approach - Contemporary focus and research issues are analyzed to provide some future directions for scholars in the field of SEA, by categorizing work in the SEA, social environmental reporting (SER, si bien no evalúa la calidad de la información (Oprisor et al., 2017).

El índice de cumplimiento fue elaborado a partir de las 8 categorías de contenidos del marco (ver anexo 1). Cada una de ellas fue una unidad de análisis.

Para cada unidad de análisis elaboramos una lista de elementos verificadores de los contenidos, obteniendo en total 42 ítems. Para establecer la puntuación utilizamos una técnica de codificación con una escala dicotómica ( 0 cuando no hay revelaciones, 1 cuando están las revelaciones).

El valor del índice se obtuvo como se muestra a continuación:

$$
I C=\frac{\sum_{i=1}^{n} a}{n}
$$

Donde,

IC: Índice de cumplimiento del ítem o unidad de análisis

n: Número de objetos de estudio (universidades)

a: Valor asignado por el cumplimiento de la divulgación del elemento

De manera similar calculamos un índice de cumplimiento para cada unidad de análisis. En nuestro caso, el instrumento de medición está influenciado por las investigaciones realizadas por Akhter e Ishihara (2018) y por Adhikariparajuli et al. (2019), ya que en ambos asignan las unidades de análisis a cada una de las categorías desarrolladas por el Marco Conceptual. 
Explorando la divulgación de información integrada por universidades colombianas...

La información de las universidades fue obtenida de la página web de cada institución, de los informes de gestión, los PEI de cada institución y adicionalmente usamos los motores de búsqueda. Resaltamos que toda la información tomada para construir el índice procede de fuentes oficiales de las universidades de la muestra. Para la última unidad de análisis, que está relacionada con las bases de preparación y presentación, tomamos el informe de rendición de cuentas o el balance social, según lo presenta cada institución del año 2019, para verificar los ítems.

\section{Resultados y discusión}

En esta sección analizamos los resultados obtenidos por cada unidad de análisis, así como un análisis detallado de las divulgaciones relacionadas con cada elemento de contenido de la lista de verificación.

\section{V.I Descripción General de la organización}

En esta unidad utilizamos seis ítems como elementos verificadores. Los resultados muestran que el índice de cumplimiento para esta unidad de análisis es del $83 \%$, con índices de cumplimiento de los seis ítems utilizados que están en el rango del $100 \%$ y el 55\%, como se puede observar en el gráfico 1.

Destaca el ítem de misión y visión, valores y cultura de la organización, tiene un índice de revelación del $100 \%$, lo que significa que todas las universidades revelan la información relacionada.

Gráfico 1. Revelación de los elementos de contenido de la unidad de análisis:

Descripción general de la organización y de su entorno externo

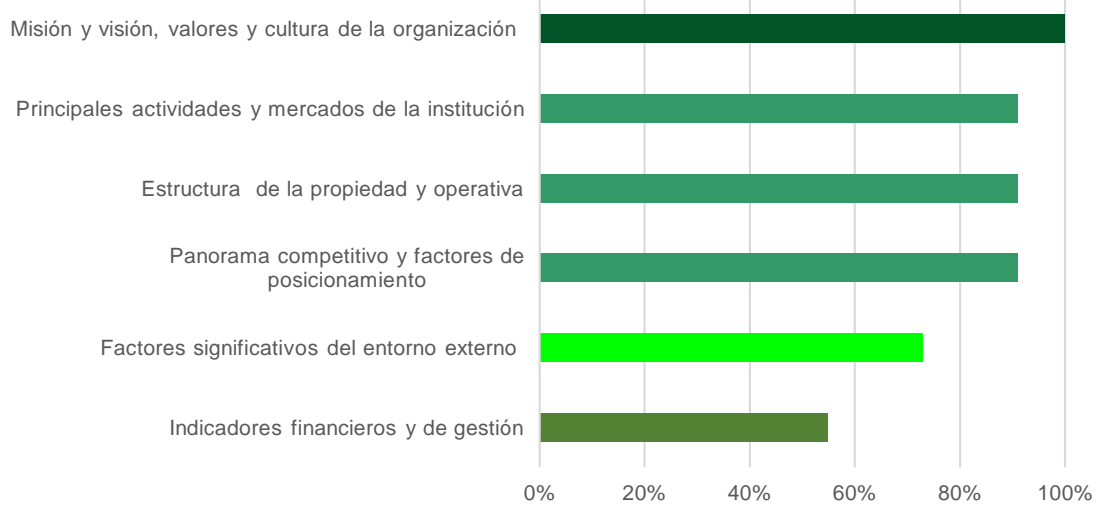

Fuente: Elaboración propia

Los elementos menos divulgados son los relativos a los Indicadores financieros y de gestión, obteniendo un índice del 53\%, lo que significa que 6 de las 11 universidades revelan información relacionada. 
Los resultados obtenidos coinciden con lo indicado por Akhter e Ishihara (2018), quienes indican que, en general las divulgaciones son altas cuando tiene que ver con información "suave" o genérica.

\section{V.2 Gobierno Corporativo}

El Marco Conceptual plantea que los IR deben ofrecer información acerca de la manera en la que el gobierno corporativo de la organización apoya la capacidad de crear valor en el corto, medio y largo plazo (IIRC, 2021), para lo cual indica que se debe revelar la estructura de liderazgo (órganos de gobierno corporativo) en la organización, los procesos específicos para la toma de decisiones estratégicas, la implementación de prácticas de gobierno corporativo que superan los requerimientos legales y la responsabilidad que asumen los órganos de gobierno para facilitar la innovación, entre otros aspectos.

En esta unidad de análisis planteamos seis elementos verificadores y obtuvimos un índice de cumplimiento general para esta unidad del 58\%. Los resultados obtenidos muestran porcentajes de divulgación para cada ítem que se sitúan entre el $100 \%$ y el $18 \%$, como se ve en el gráfico 2 .

Gráfico 2. Revelación de los elementos de contenido de la unidad de análisis: Gobierno Corporativo.

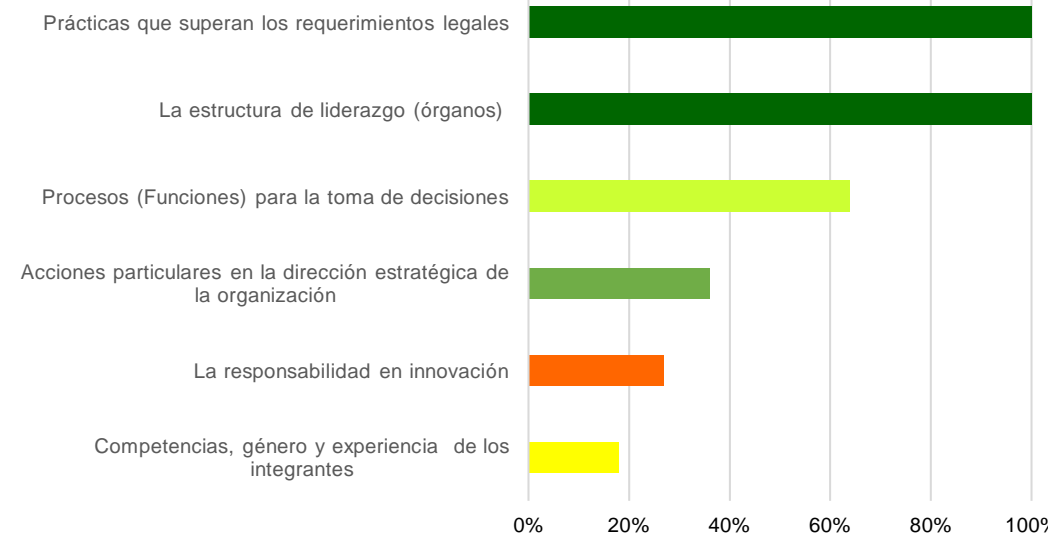

Fuente: Elaboración propia

Los ítems más divulgados en esta unidad de análisis son los relativos a la implementación de prácticas sostenibles que superan los requerimientos legales y a la revelación de la estructura de liderazgo, que alcanzan un índice de divulgación del $100 \%$. Así, por ejemplo, para 2019, las universidades Nacional y Externado de Colombia están comprometidas con el cumplimiento de los Objetivos de Desarrollo Sostenible (ODS - 2030); mientras que las universidades del Rosario (2019),del Norte (2019) y EAFIT (2019) están alineadas con los principios del Pacto Global. La 
Explorando la divulgación de información integrada por universidades colombianas...

Universidad del Norte, publica por primera vez el informe bajo el estándar de del sistema de indicadores de responsabilidad social universitaria del Pacto Global. La Universidad EAFIT, además, por cuarto año publica el informe de sostenibilidad bajo la metodología GRI. Por otra parte, las Universidades del Norte, Nacional y del Rosario están dentro de las cien más sostenibles del mundo de acuerdo con el UI Greenmetric World University Ranking (Semana, 2019). Navarrete Oyarce and Gallizo Larraz (2019) señalan al respecto que se percibe positivamente a las organizaciones que revelan información no financiera.

El aspecto en que menos información se revela es en la divulgación de las competencias (formación), género (o nombre) y experiencia de la estructura de liderazgo de la organización con solo un $18 \%$. Esto indica que solo dos Universidades -Del Rosario y Del Valle- hacen revelaciones completas al respecto.

\section{V.3 Modelo de Negocio}

El modelo de negocio es un sistema de transformación de insumos cuyo objetivo es cumplir con los propósitos estratégicos de la organización y la creación de valor a corto, medio y largo plazo (IIRC, 2021). Se incluyeron seis elementos verificadores relacionados con las principales actividades de la organización y se obtuvo un índice de cumplimiento de $91 \%$ para esta unidad de análisis, con índices parciales de divulgación situados entre el $100 \%$ y el $82 \%$, como se muestra en el gráfico 3. Este resultado concuerda con el estudio realizado por Mauro et al. (2020), teniendo en cuenta que dada su naturaleza las universidades divulgan la información relacionada con las inversiones y resultados obtenidos en innovación, patentes y en general con la investigación.

Gráfico 3. Revelación de los elementos de contenido de la unidad de análisis:

Modelo de Negocio. Revela los impactos sociales y medioambientales
de las actividades

Revelan información relacionada con egresados

Específica las principales actividades

Divulga la mejora de procesos, capacitación a empleados

Relacionan las principales actividades con el uso de los capitales

Señala la lista de las principales fuentes de ingresos

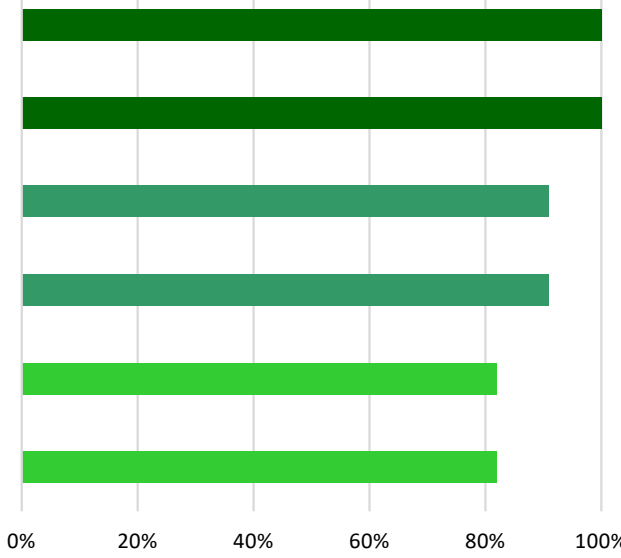

Fuente: Elaboración propia 
Todas las universidades de la muestra revelan la información relacionada con los egresados y con los impactos sociales y medioambientales de las actividades de la institución, por lo tanto, el índice de divulgación fue del 100\% para esos ítems.

Así mismo, los menores índices de divulgación de esta unidad son los relacionados con la revelación de las principales actividades de la organización con el uso de los capitales de la organización y la lista de las principales fuentes de ingresos, con un resultado del $82 \%$ de divulgación en cada uno de ellos.

\section{V.4 Riesgos y Oportunidades}

El Marco Conceptual de la IR también señala la necesidad de divulgar información acerca de los riesgos y las oportunidades específicas que afectan la capacidad de la organización para crear valor en el corto mediano y largo plazo (IIRC, 2021). Para ello, en esta unidad de análisis utilizamos 3 ítems que incluyen la revelación de la fuente de riesgos y oportunidades específicos para la organización, la evaluación que hace la organización sobre la probabilidad que el riesgo suceda y las medidas específicas que hace la organización para mitigar o gestionar los riesgos. Esta unidad de análisis obtuvo un índice de revelación de $58 \%$.

Gráfico 4. Revelación de los elementos de contenido de la unidad de análisis:

Riesgos y oportunidades

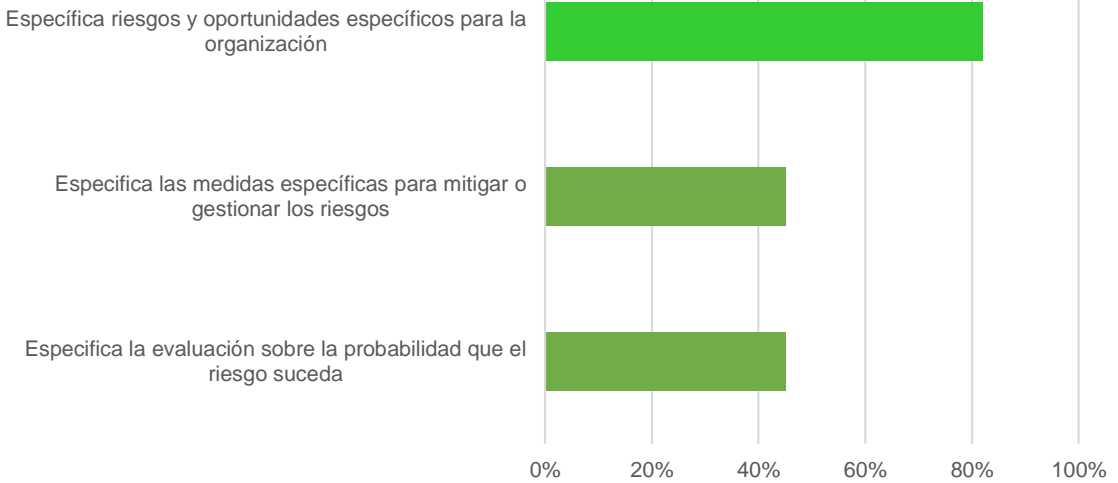

Fuente: Elaboración propia

Como se puede observar en el gráfico 4 , la información relativa a riesgos y oportunidades para la organización alcanza un $82 \%$ de divulgación, es decir que solo dos universidades de la muestra -De la Sabana y Externado- no revelan información al respecto en la web.

Para los demás ítems, el índice de revelación obtenido es del 45\%, encontrando que las Universidades de Antioquia, Industrial de Santander, Nacional de Colombia y del Valle, revelan la información solicitada (todas de carácter público) mediante matrices de riesgo. La Universidad del Norte revela el proceso de 
Explorando la divulgación de información integrada por universidades colombianas...

evaluación de riesgos en el informe de sostenibilidad, las demás universidades privadas no revelan información relacionada con esta unidad de análisis.

Estos resultados están en línea con el estudio de Pwc 2013, citado por de Villiers et al.(2014) realizado en empresas de los países Bajos, que identificaron la comunicación de los principales riesgos y oportunidades de mejora, como una de las áreas más críticas ya que solo el 3\% de las empresas comunicaban dicha información.

\section{V.5 Estrategia y asignación de recursos}

El Marco Conceptual de la IR señala la necesidad de divulgar información acerca de los objetivos estratégicos de la organización a corto, mediano y largo plazo; las estrategias de la organización relacionadas con estos objetivos; los planes de asignación de recursos para poner en marcha la estrategia; entre otros (IIRC, 2021).

El índice de divulgación global obtenido en esta unidad de análisis es del 62\%. Respecto a los 6 elementos verificadores utilizados (ver gráfico 5), el item de mayor divulgación es el de revelación de los objetivos estratégicos de la organización a corto, mediano y largo plazo, con un índice de revelación del 91\%, por cuanto en el PEI todas las universidades hacen un ejercicio prospectivo en un plazo de 4 años. Sin embargo, el PEI de la Universidad Externado de Colombia no se encuentra publicado en la web.

Gráfico 5. Revelación de los elementos de contenido de la unidad de análisis:

Estrategia y asignación de recursos.

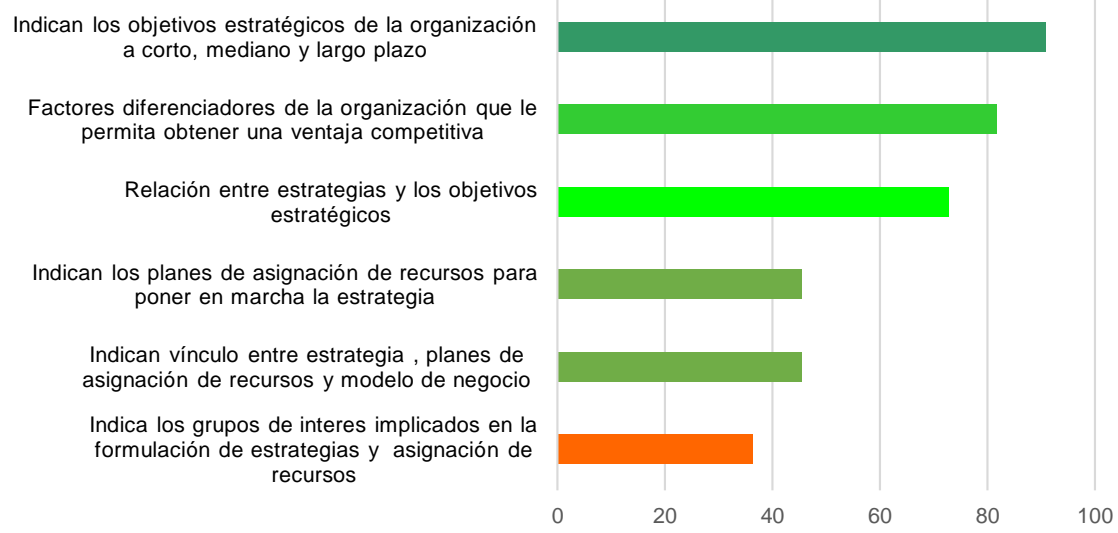

Fuente: Elaboración propia

Por el contrario, los índices con menor divulgación son los relacionados con la revelación de los grupos de interés implicados en la formulación de estrategias y de la asignación de recursos, con un 36\%. En los ítems de los planes de 
asignación de recursos para poner en marcha la estrategia y el vínculo entre la estrategia de la organización, los planes de asignación de recursos y el modelo de negocio de la universidad, el índice alcanzó el $45 \%$, es decir que en cada uno de esos ítems cinco de las seis universidades revelan información. Al respecto, algunos autores como De Villiers et al. (2014); y Navarrete et al. (2019), han señalado posibles problemas de agencia, teniendo en cuenta que quienes preparan la información son los administradores de las organizaciones.

\section{V.6 Desempeño}

El desempeño debe contener información cualitativa y cuantitativa (IIRC, 2013), por lo que incluimos 5 elementos verificadores: los indicadores cuantitativos con respecto a metas, riesgos y oportunidades; los efectos de la organización en los capitales; el estado de la relación con los grupos de interés y la respuesta de la organización a las necesidades de los grupos de interés; el vínculo entre el desempeño anterior, el actual y el esperado; los indicadores que vinculen medidas financieras con otras medidas o narrativas que expliquen las implicaciones con los capitales o con otras relaciones causales.

El resultado global fue del 36\%para esta unidad de análisis; con índices para cada ítem situados entre el $27 \%$ y el $45 \%$, como se muestra en el gráfico 6 .

Específicamente, un índice de revelación del 45\% fue evidenciado para dos de los ítems, el estado de la relación con los grupos de interés y para la respuesta de la organización a las necesidades de estos y para el vínculo entre el desempeño anterior, el actual y el esperado.

Gráfico 6. Revelación de los elementos de contenido de la unidad de análisis: Desempeño.

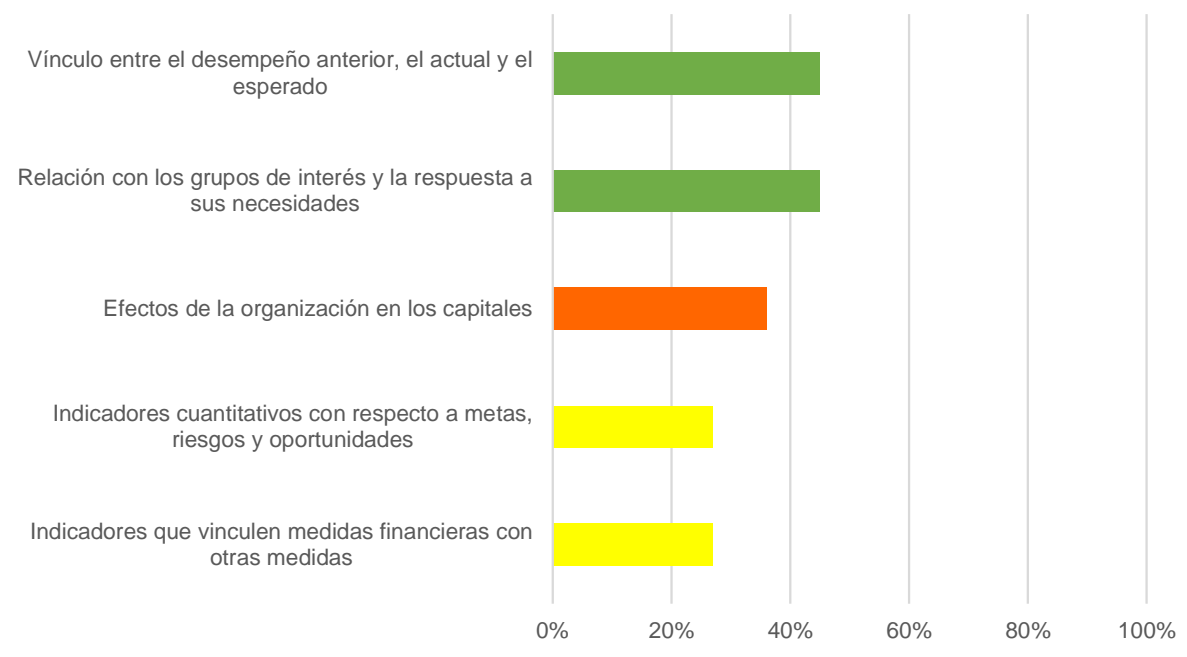

Fuente: Elaboración propia 
Explorando la divulgación de información integrada por universidades colombianas...

Los ítems donde obtuvimos menor índice de divulgación son en los indicadores tanto cuantitativos con respecto a metas, riesgos y oportunidades, como en los que vinculan medidas financieras con otras oportunidades, donde obtuvimos un índice de revelación de $27 \%$ en cada uno, solo 3 de las once universidades publican información relacionada. Los resultados van en contravía de los obtenidos en las universidades italianas por Mauro et al. (2020), evidenciando diferencias entre los reportes presentados por las universidades italianas y colombianas objeto de los estudios y abriendo el camino para posibles estudios futuros.

\section{V.7 Perspectivas futuras}

En este aspecto incluimos 6 elementos verificadores: las expectativas de la organización sobre el entorno externo, la manera en que la organización planea enfrentar los posibles cambios esperados, las posibles implicaciones en el financiamiento futuro y de disponibilidad de otros capitales, la revelación de indicadores de fuentes externas reconocidas con información relevante, la revelación de la comparación del desempeño actual con los objetivos fijados y la revelación de los requisitos legales que la organización debe cumplir. Para esta unidad de análisis obtuvimos un índice de revelación global del 48\%, los resultados obtenidos se evidencian en el gráfico 7 . Este resultado concuerda con lo indicado por Correa-García et al. (2017), teniendo en cuenta que las organizaciones reconocen como una desventaja competitiva revelar información que puede considerarse beneficiosa para la competencia.

Gráfico 7. Revelación de los elementos de contenido de la unidad de análisis:

Perspectivas futuras

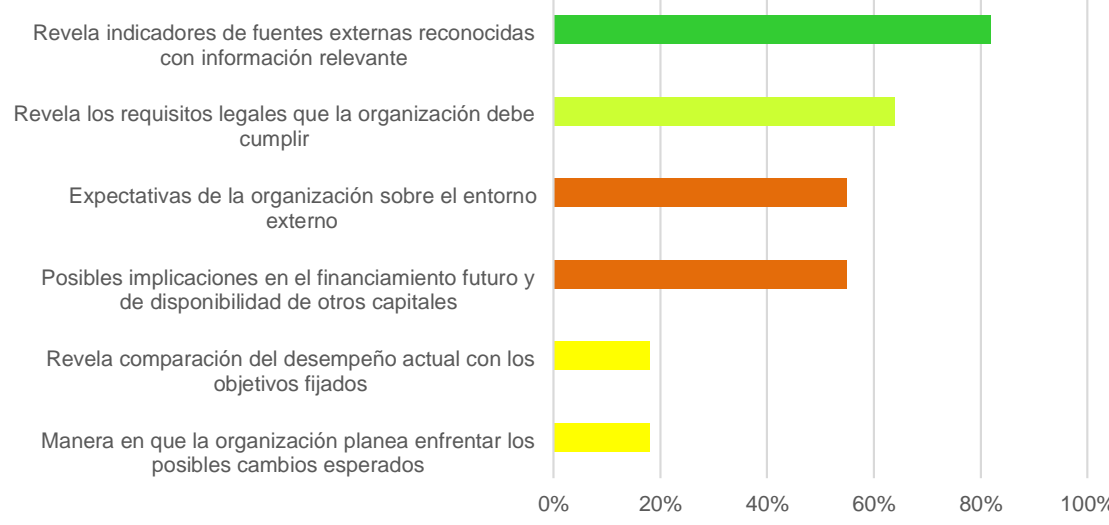

Fuente: Elaboración propia

Los resultados muestran un índice de revelación del $82 \%$ con respecto a la divulgación de indicadores de fuentes externas reconocidas con información relevante. Dos de las universidades -Nacional y Externado- no revelan información al respecto. 
Los resultados muestran los menores índices de revelación en dos ítems: la manera en que la organización planea enfrentar los posibles cambios esperados y en la revelación de la comparación del desempeño actual con los objetivos fijados, donde en cada uno de dichos ítems obtuvimos un índice de revelación de $18 \%$, es decir que en cada uno de dichos ítems dos de las universidades de la muestra revelan información relacionada.

\section{V.8 Bases para la elaboración y presentación}

De acuerdo con el Marco de la IR, las organizaciones deben revelar información relacionada con la descripción sobre la determinación de la materialidad, aplicada al reporte y como fue determinada; los marcos y métodos significativos utilizados para cuantificar o evaluar asuntos materiales; la identificación del papel de los órganos de gobierno corporativo en el informe y la dentificación de los involucrados en la preparación y revisión del informe. Esta unidad de análisis obtuvo un índice de revelación del $50 \%$. Este resultado es coincidente con el de Mauro et al. (2020), donde el análisis de este contenido obtuvo el menor índice de revelación.

Los índices por cada elemento se muestran en el gráfico 8 y reflejan que el ítem de menos divulgación es el relacionado con la determinación de la materialidad aplicada al reporte, en el que solo dos universidades revelan informaciónEAFIT y Del Norte-.

Gráfico 8. Revelación de los elementos de contenido de la unidad de análisis: Bases para la preparación y presentación.

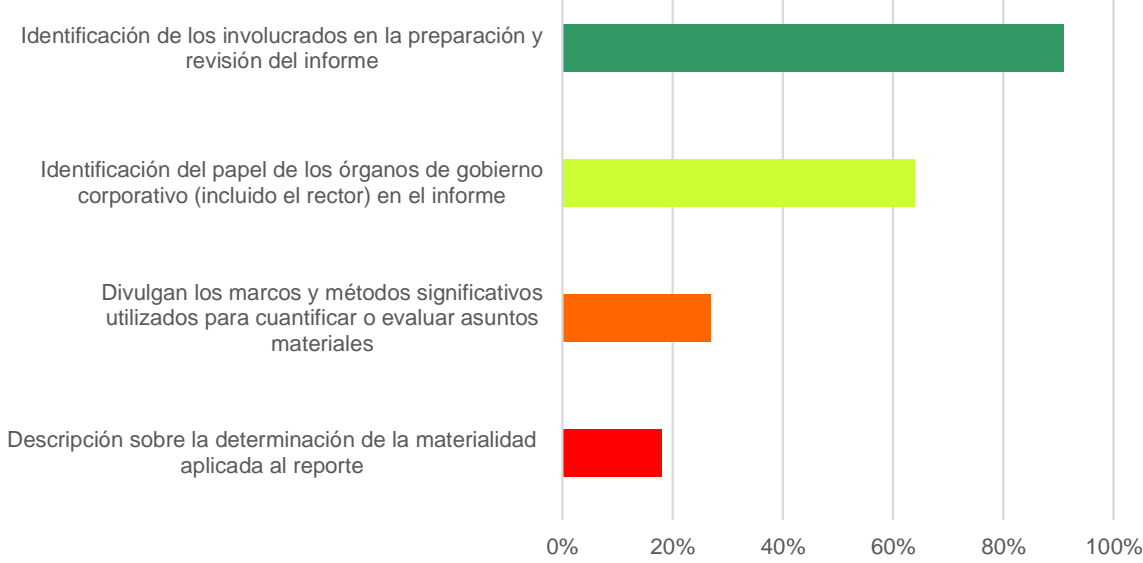

Fuente: Elaboración propia

Como resultado general, podemos ver que los porcentajes de divulgación en términos globales de los bloques fundamentales de información que recoge el 
Explorando la divulgación de información integrada por universidades colombianas...

marco conceptual (unidades de análisis) son dispares, situándose entre el 91\% y el 36\%. Los contenidos relacionados con la descripción general de la organización y con el modelo de negocio son los que más revelaciones tienen, mientras que el desempeño es el que menos, como se aprecia en el gráfico 9. Estos resultados son coherentes con el estudio realizado en Universidades Italianas realizado por Mauro et al. (2020), donde los resultados muestran un nivel de divulgación entre un $80 \%$ y un $43 \%$ en los ítems analizados.

En cuanto a información relacionada con el desempeño, solo tres de las universidades de la muestra hacen revelaciones sobre indicadores tanto cuantitativos con respecto a las metas, riesgos y oportunidades como los que vinculan medidas financieras con otras medidas o narrativas que expliquen las implicaciones con los capitales o con otras relaciones causales, lo cual no permite tener una medida del desempeño de las organizaciones.

Con respecto al Modelo de Negocio, en dos de los ítems verificados todas las universidades hacen revelaciones, que son en los relacionados con las ventajas o acuerdos para los egresados y en cuanto a la revelación de los impactos sociales y medioambientales de las actividades de la institución.

Gráfico 9. Revelación de los resultados por unidad de análisis.

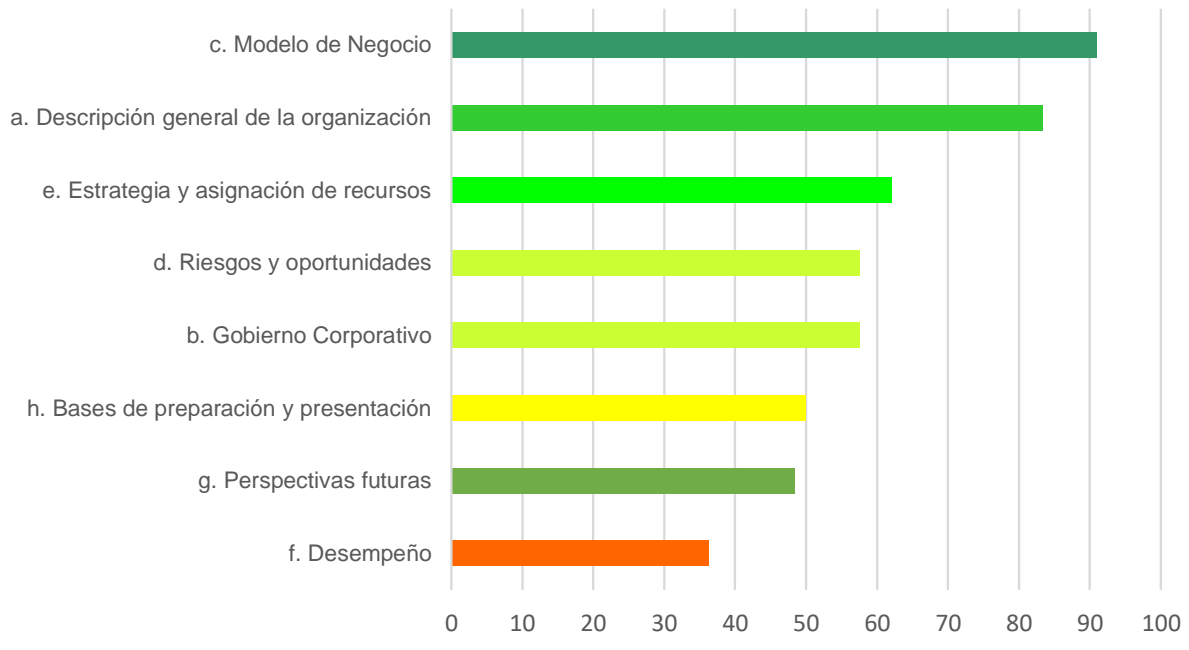

Fuente: Elaboración propia

\section{V.9 Resultados por universidad}

Aunque el objetivo principal de nuestro estudio no fue hacer un análisis de la divulgación de información por universidades, consideramos pertinente mostrar los resultados obtenidos en este sentido. 
Como se puede ver en el gráfico 10, la universidad que más realiza la divulgación solicitada, de acuerdo con el Marco Conceptual es la Universidad de Antioquia, que obtuvo un índice del $90 \%$. Sin embargo, la información que divulga en internet no es completa en cuanto a gobierno corporativo, pues no da conocer completamente la estructura de liderazgo (formación, género y experiencia), los procesos específicos para la toma de decisiones estratégicas y las acciones de los órganos de gobierno en cuanto a la dirección estratégica de la organización. De la misma manera no se pudo verificar la descripción sobre la determinación de la materialidad aplicada al reporte.

Gráfico 10. Divulgación de la información revelada clasificada por universidades.

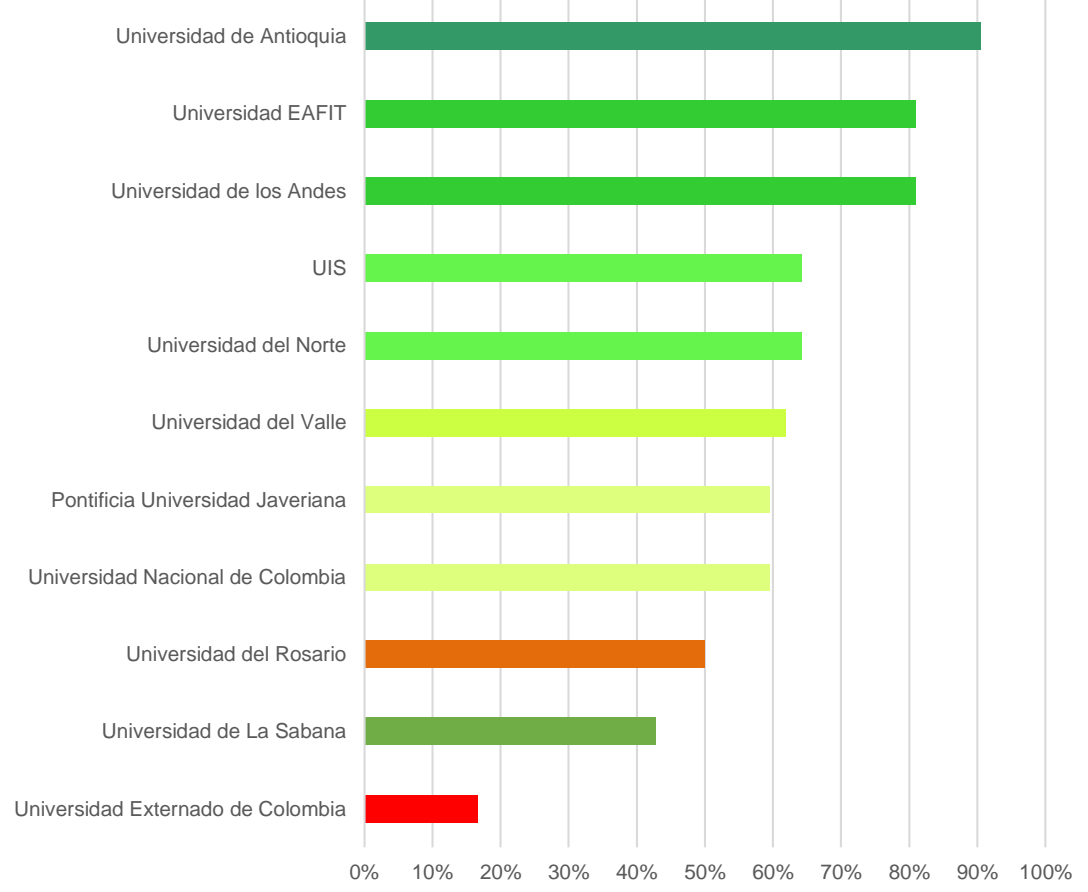

Fuente: elaboración propia

Siete de las diez universidades de la muestra obtuvieron un puntaje en los índices de divulgación de información entre el $81 \%$ y el 60\%, que son las Universidades de los Andes, EAFIT, del Norte, UIS, del Valle, Nacional y Javeriana. De estas universidades todas presentan informe de rendición de cuentas o balance social en versiones descargables en formato pdf, con excepción de la universidad EAFIT, que presenta la rendición de cuentas en dos documentos en formato electrónico en su página web. Dado que este grupo de universidades cuenta con porcentajes superiores en el índice de cumplimiento, sería pertinente indagar 
en futuros estudios sobre la proclividad de estas organizaciones a implementar el pensamiento integrado como un proceso estratégico que les permita más adelante ser pioneras en la emisión de un informe integrado que cumpla con las directrices del IIRC.

Solo tres de las universidades de la muestra, obtuvieron índices de divulgación inferiores o igual al 50\%, que son las Universidades Rosario, Externado y de la Sabana, con índices de divulgación que están entre el 50\% y el $17 \%$.

El índice global de información divulgada en internet por la Universidad Externado de Colombia fue del $17 \%$, teniendo en cuenta que de los 42 ítems solo publica información en 12 de ellos. En las unidades de análisis de riesgos y oportunidades y en la de perspectivas futuras, no fue posible encontrar información relacionada con ninguno de los ítems, por lo que los índices de revelación obtenidos en estos apartados fueron del $0 \%$.

Como un punto adicional, vale la pena llamar la atención sobre la fragmentación de información dispuesta por las universidades estudiadas. A pesar de que muchas de estas cuentan con varios de los contenidos necesarios para la elaboración de un informe integrado, es pertinente resaltar las limitaciones que esta dispersión podría generar para sus grupos de interés y para la construcción de un pensamiento integrado.

Tal como ha sido señalado por Feng et al. (2017) no existen precedentes claros de integración en el contexto de los reportes, sin embargo, una cuestión crucial para materializar la integración de información reside en que primero se realice un proceso interno en el que la toma de decisiones esté permeada por una conciencia holística de la organización, de sus grupos y de sus capitales. Así, los resultados de este estudio reflejan una oportunidad para que estas universidades implementen el pensamiento integrado de cara a realizar transformaciones estratégicas y prácticas. Adicionalmente, en el caso de las universidades con los índices de cumplimiento más altos, sería importante la aplicación del principio de conectividad (Rivera-Arrubla y Zorio-Grima, 2016) that is, the Integrated Report (IR que haría que, sin sacrificar la profundidad de la información, pudiera entregarse un informe conciso y acorde con las expectativas de los stakeholders.

\section{VI.Conclusiones}

Teniendo en cuenta la cantidad de información que se divulga en la actualidad, tanto de carácter financiero como no financiero, el objetivo de nuestro estudio fue analizar en qué medida la información solicitada para construir un reporte integrado, de acuerdo con los requerimientos del Marco Conceptual del IIRC, ya está revelada en internet por parte de las universidades colombianas presentes en el ranking QS 2019. 
Los resultados mostraron que el grado de divulgación de la información que debería contener un IR es heterogéneo en la muestra. Así pues, los índices de divulgación en las ocho unidades de análisis que van desde el $91 \%$ de cumplimiento para la información relacionada con el modelo de negocio, hasta el 36\% para los datos relacionados con el desempeño. Los contenidos relacionados con el modelo de negocio y la descripción general de la organización son los más divulgados, mientras que la relativa al gobierno corporativo y los riesgos y oportunidades alcanzan un 58\%. Otras informaciones donde será necesario realizar avances en la divulgación son las relacionadas con las bases para la preparación y presentación (50\%), perspectivas futuras (48\%) y el desempeño (36\%).

Si nos detenemos en el detalle de la información divulgada, solo en 5 de los 42 ítems todas las universidades de la muestra revelan la información solicitada: la misión y visión, valores y cultura de la organización; la estructura de liderazgo en la organización; las prácticas de gobierno corporativo que superan los requerimientos legales; los acuerdos o ventajas para los egresados de la organización y revela los impactos sociales y medioambientales de las actividades de la institución. Aunque, vale la pena señalar que dicha información se encuentra dispersa en varios links e informes de las instituciones.

Los ítems donde menos información divulgada encontramos son los relacionados con la divulgación de las competencias de la estructura de liderazgo de la organización; la manera en que la organización planea enfrentar los posibles cambios esperados; la comparación del desempeño actual con los objetivos fijados y la determinación de la materialidad aplicada al reporte, donde solo dos universidades revelan información.

Podemos concluir que, una parte importante de la información necesaria para la elaboración de un IR ya se encuentra disponible en la mayoría de universidades colombianas, por lo que sería necesario avanzar en dos direcciones. Por una parte, desarrollar y mejorar aquellos aspectos menos divulgados y por otro reunir la información dispersa a lo largo de diferentes informes, organizándola de acuerdo con lo establecido en el marco conceptual de la IR. Es importante destacar que las universidades objeto de la muestra hacen ejercicios de prospección en lo relacionado con las perspectivas futuras y la estrategia, mediante el PEI, en el cual plasman los objetivos que se planean alcanzar en un plazo de cuatro años; de la misma manera que hacen rendición de cuentas y presentan información no solamente financiera cada año.

En cuanto al futuro en el campo de investigación de los IR y principalmente en referencia al ámbito de aplicación, sería necesario analizar si la adaptación del Marco conceptual es adecuado en todo caso, como en entidades del sector público o sin ánimo de lucro. En cuanto al campo empírico, será necesario realizar estudios encaminados a comprobar la mejora en las organizaciones a partir 
Explorando la divulgación de información integrada por universidades colombianas...

de la implementación de los IR, así como los distintos factores que pueden influir en dicha implementación.

Finalmente, como limitaciones de este estudio señalamos su carácter exploratorio y el alcance mismo del objetivo, que no procuró realizar un examen a los informes integrados de las universidades, sino que, atendiendo al estado incipiente de su implementación se enfocó en observar qué contenidos de los dispuestos en el marco IIRC se encuentran ya divulgados y están disponibles en la web. De igual forma, resaltamos que los resultados y conclusiones de este estudio se circunscriben únicamente al grupo de universidades estudiadas.

\section{Referencias bibliográficas}

Adams, S., y Simnett, R. (2011). Integrated Reporting: An Opportunity for Australia's Notfor-Profit Sector. En Australian Accounting Review (Vol. 21, Número 3, pp. 292-301). https://doi.org/10.1111/j.1835-2561.2011.00143.x

Adhikariparajuli, M., Hassan, A., Fletcher, M., y Elamer, A. (2019). Integrated reporting in UK higher education institutions. Sustainability Accounting, Management and Policy Journal, ahead-of-p(ahead-of-print). https://doi.org/10.1108/sampj-03-2018-0093

AECA. (2011). HACIA LA INFORMACIÓN INTEGRADA La comunicación de valor en el Siglo XXI. 51.

Akhter, T., y Ishihara, T. (2018). Assessing the Gap Between Integrated Reporting and Current Corporate Reporting: A Study in the UK. International review of business, 18(March), 137-157. https://doi.org/10.13140/RG.2.2.30407.91048

Bernardi, C. (2020). Leonardo: All that Glitters Is not Gold. En C. Bernardi (Ed.), Implementing Integrated Reporting (SpringerBr, pp. 37-55). Springer. https://doi.org/10.1007

Brusca, I., y Labrador, M. (2017). El Marco Conceptual De La Información Integrada Y Su Aplicación En El Sector Público. Revista Española De Control Externo, XIX(Septiembre), 13-38.

Brusca, I., Labrador, M., y Larran, M. (2018). The challenge of sustainability and integrated reporting at universities: A case study. Journal of Cleaner Production, 188, 347-354. https://doi.org/10.1016/j.jclepro.2018.03.292

Churet, C., y Eccles, R. G. (2014). Integrated Reporting, Quality of Management, and Financial Performance. Journal of Applied Corporate Finance, 26(1), 55-65. https://doi. org/10.1111/jacf.12054

Ley 872 de diciembre 30 de 2003, 2003 Diario Oficial 24 (2003).

Ley 1712 de 6 de marzo de 2014, Diario Oficial 57 (2014).

Correa-García, J. A., Hernández-Espinal, M. C., Vásquez-Arango, L., y Soto-Restrepo, Y. M. (2016). Reportes integrados y generación de valor en empresas colombianas incluidas en el Índice de Sostenibilidad Dow Jones. Cuadernos de Contabilidad, 17(43), 73-108. https://oi.org/10.11144/javeriana.cc17-43.rigv

de Villiers, C., Rinaldi, L., y Unerman, J. (2014). Integrated reporting: Insights, gaps and an agenda for future research. Accounting Auditing and Accountability Journal, 27(7). https://doi.org/10.1108/AAAJ-06-2014-1736 
de Villiers, C., Venter, E. R., y Hsiao, P. C. K. (2016). Integrated reporting: background, measurement issues, approaches and an agenda for future research. Accounting and Finance, 57(4), 937-959. https://doi.org/10.1111/acfi.12246

Dumay, J., Bernardi, C., Guthrie, J., y Demartini, P. (2016). Integrated reporting: A structured literature review. Accounting Forum, 40(3), 166-185. https://oi.org/10.1016/j. accfor.2016.06.001

EAFIT. (2019). EAFIT - PACTO GLOBAL. http://www.eafit.edu.co/pactoglobal/Paginas/eafitpacto-global.aspx.

Feng, T., Cummings, L., y Tweedie, D. (2017). Exploring integrated thinking in integrated reporting - an exploratory study in Australia. Journal of Intellectual Capital, 18(2), 330353. https://doi.org/10.1108/JIC-06-2016-0068

Galeano, M. E. (2004). Diseño de proyectos en la investigación cualitativa. Fondo Editorial Universidad EAFIT.

Gallego-Álvarez, I., Rodríguez-Domínguez, L., y García-Sánchez, I. M. (2011). Information disclosed online by Spanish universities: Content and explanatory factors. Online Information Review, 35(3), 360-385. https://doi.org/10.1108/14684521111151423

Giraldo-López, J., Soto-Sossa, M., y Mejía-Delgado, J. A. (2018). Características de la información contenida en los reportes integrados: una visión para el mercado integrado de américa latina (MILA) en el 2014. Contaduría Universidad de Antioquia, 163-198. https://doi.org/10.17533/udea.rc.n73a07

Gómez-Villegas, M. (2017). El Reporte Integrado en el Sector Público: Una mirada desde Latinoamérica. Revista Española de Control Externo, XiX(57), 67-92.

GRI. (2021). SDD Database. Sustainability Disclosure Database. https://database. globalreporting.org/search/

Guthrie, J., y Abeysekera, I. (2006). Content analysis of social, environmental reporting: what is new? Journal of Human Resource Costing \& Accounting, 10(2), 114-126. https:// doi.org/10.1108/14013380610703120

Guthrie, J., Domingues, A. R., Manes-Rossi, F., y Orelli, R. (2020). Integrated reporting and sustainable development goals in universities. En The Routledge Handbook of Integrated Reporting (pp. 419-439). Routledge.

Humphrey, C., O'dwyer, B., y Unerman, J. (2017). Re-theorizing the configuration of organizational fields: the IIRC and the pursuit of «Enlightened» corporate reporting. Accounting and Business Research, 47(1), 30-63. https://doi.org/10.1080/00014788.201 6.1198683

Iacuzzi, S., Garlatti, A., Fedele, P., y Lombrano, A. (2020). Integrated reporting and change: evidence from public universities. Journal of Public Budgeting, Accounting and Financial Management, 32(2), 291-310. https://doi.org/10.1108/JPBAFM-08-2019-0120

IIRC. (2012). TOWARDS INTEGRATED REPORTING. Communicating Value in the 21st Century Summary of Responses to the September 2011 Discussion Paper and Next Steps. www. theiirc.org/the-integrated-reporting-discussion-paper/discussion-paper-submissions/

IIRC. (2013). El Marco Conceptual Internacional de información Integrada.

IIRC. (2021). < IR > FRAMEWORK (Número January). https://integratedreporting.org/wpcontent/uploads/2021/01/InternationallntegratedReportingFramework.pdf 
Explorando la divulgación de información integrada por universidades colombianas...

IIRC, I. I. R. C. (2019). IR Reporters. IR Reporters.

Lee, K. W., y Yeo, G. H. H. (2016). The association between integrated reporting and firm valuation. Review of Quantitative Finance and Accounting, 47(4), 1221-1250. https://doi. org/10.1007/s11156-015-0536-y

Lodhia, S., y Stone, G. (2017). Integrated Reporting in an Internet and Social Media Communication Environment: Conceptual Insights. Australian Accounting Review, 27(1), 17-33. https://doi.org/10.1111/auar.12143

Low, M., Samkin, G., y Li, Y. (2015). Voluntary reporting of intellectual capital: Comparing the quality of disclosures from New Zealand, Australian and United Kingdom universities. Journal of Intellectual Capital, 16(4), 779-808. https://doi.org/10.1108/JIC03-2015-0022

Macias, H. A., y Farfan-Lievano, A. (2017). Integrated reporting as a strategy for firm growth: multiple case study in Colombia. Meditari Accountancy Research, 25(4), 605628. https://doi.org/10.1108/MEDAR-11-2016-0099

Mauro, S. G., Cinquini, L., Simonini, E., y Tenucci, A. (2020). Moving from social and sustainability reporting to integrated reporting: Exploring the potential of Italian public-funded universities' reports. Sustainability (Switzerland), 12(8), 3172. https:// doi.org/10.3390/SU12083172

Navarrete Oyarce, J., y Gallizo Larraz, J. (2019). Reporte Integrado como herramienta de transparencia empresarial. Revista academia y negocios, 4(2), 71-82.

Oprisor, T., Cordos, George-Silviu; Crisan, A.-R., Nistor, Cristina Silvia; Stefanescu, C. A., y Tiron-Tudor, A. (2017). The emergence of integrated reporting in public higher education: evidence from existing disclosure practice. 40 Congreso de la European Accounting Association Anual, 1-35.

Decreto 2375 de 17 de julio de 2006, Diario Oficial 1 (2006).

Reuter, M., y Messner, M. (2015). Lobbying on the integrated reporting framework: An analysis of comment letters to the 2011 discussion paper of the IIRC. En Accounting, Auditing and Accountability Journal (Vol. 28, Número 3). https://doi.org/10.1108/AAAJ03-2013-1289

Richardson, A. J., y Kachler, M. D. (2017). University sustainability reporting: A review of the literature and development of a model. Handbook of Sustainability in Management Education: In Search of a Multidisciplinary, Innovative and Integrated Approach, 385-405. https://doi.org/10.4337/9781785361241.00027

Rivera-Arrubla, Y. A., y Zorio-Grima, A. (2016). Integrated Reporting, Connectivity, and Social Media. Psychology and Marketing, 33(12), 1159-1165. https://doi.org/10.1002/ mar.20953

Rivera-Arrubla, Y. A., Zorio-Grima, A., y García-Benau, M. A. (2016). El concepto de informe integrado como innovación en reporting corporativo. Journal of Innovation $\mathcal{E}$ Knowledge, 1(3), 144-155. https://doi.org/10.1016/j.jik.2016.01.016

Rodríguez, R. A. (2019). Gestión de la información cuantitativa en las universidades Pistas para su abordaje en la era de la sobreinformación.

Rowbottom, N., y Locke, J. (2015). The Emergence of Integrated Reporting. Accounting and Business Research. 
Scopus. (2021). Analyze search results. https://www-scopus-com.ezproxy.unal.edu.co/ term/analyzer.uri?sid $=8 \mathrm{f} 7818 \mathrm{f} 05331 \mathrm{db} 1 \mathrm{fb} 577540867 \mathrm{~d} 80 \mathrm{e} 02 \&$ origin $=$ result slist $\& s r c=s \& s=$ TITLE-ABS-KEY\%2528Integrated + reporting $\% 2529 \&$ sort $=$ plf$\mathrm{f} \& \mathrm{sdt}=\mathrm{cl} \& \mathrm{sot}=\mathrm{b} \& \mathrm{sl}=35 \& \mathrm{count}=3755 \&$ analyzeResults $=$ Analyze + results\&cluster $=$ scopubyr $\% 252$

Semana. (2019). Las estrategias que tienen a 5 universidades nacionales en el top 100 de las más sostenibles del mundo. revista Semana.

Solomon, J., y Maroun, W. (2012). Integrated reporting: the influence of King III on social , ethical and environmental reporting. En The Association of Chartered Certified Accountants, .

Tascon, M. T., Eira, S., y Rio, R. (2015). La creación de valor del Integrated Reporting. $V$ Congresso dos TOC.

Toro Jaramillo, I. D., y Parra Ramírez, R. D. (2010). Fundamentos epistemológicos de la investigación y la metodología de la investigación cualitativa/cuantitativa. Fondo Editorial Universidad EAFIT.

Universidad del Norte. (2019). Informe de sostenibilidad 2019.

University of The Free State. (2018). University of The Free State Annual Report 2018. En AIMS Mathematics (Vol. 1, Número 1).

Veltri, S., y Silvestri, A. (2015). The Free State University integrated reporting: a critical consideration. Journal of Intellectual Capital, 16(2), 443-462. https://doi.org/10.1108/ JIC-06-2014-0077 
Explorando la divulgación de información integrada por universidades colombianas...

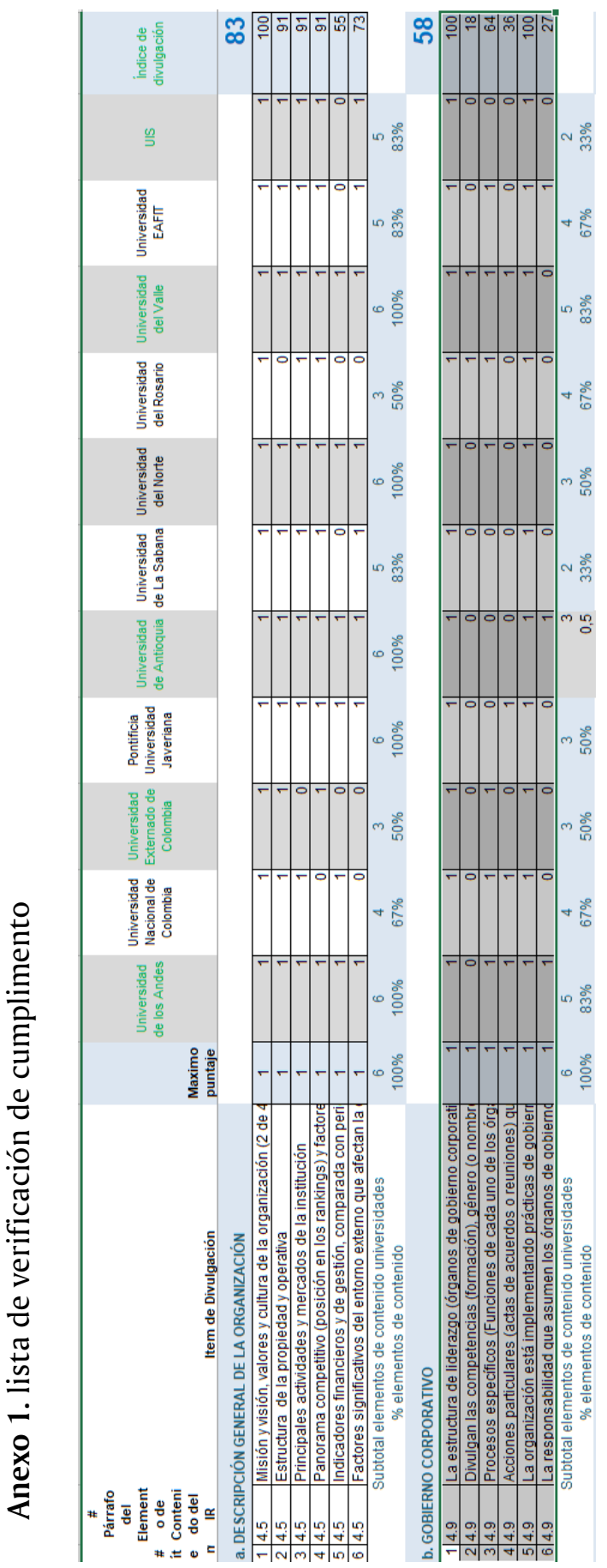




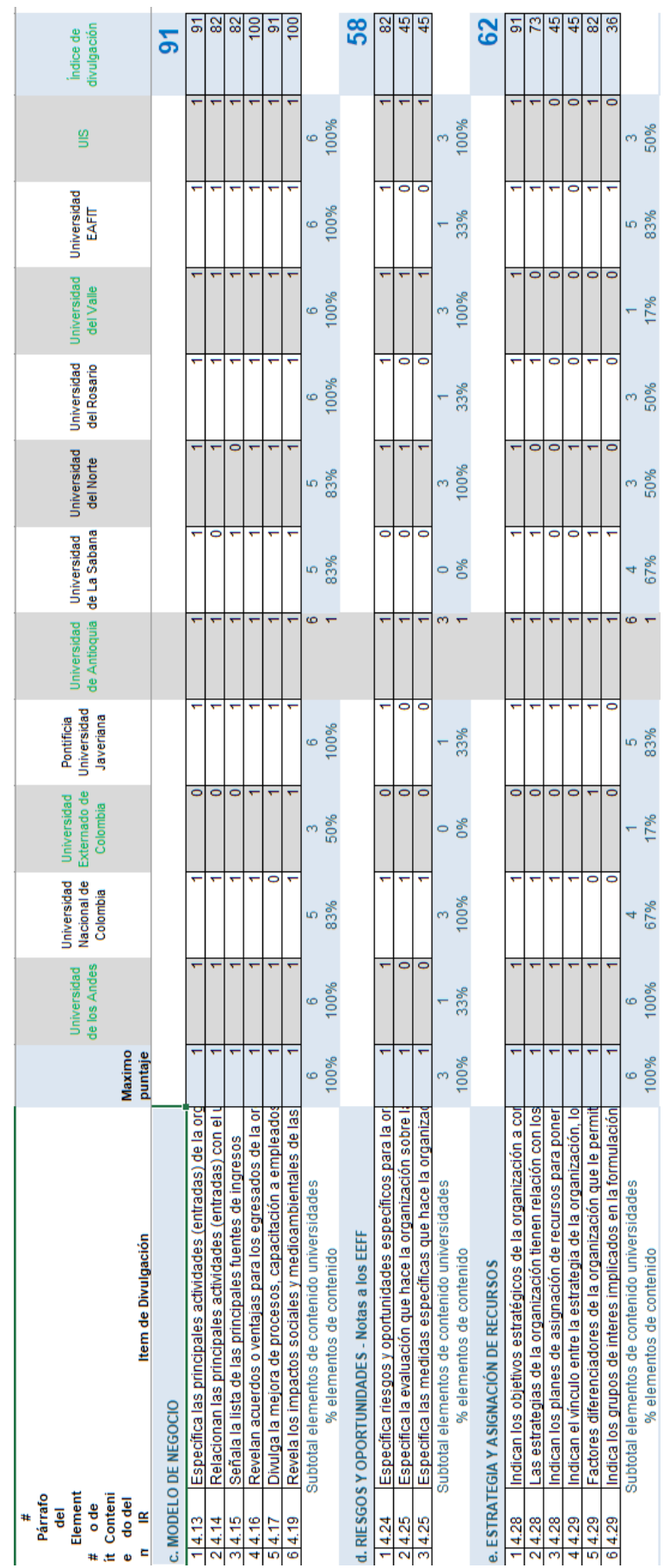


Explorando la divulgación de información integrada por universidades colombianas...

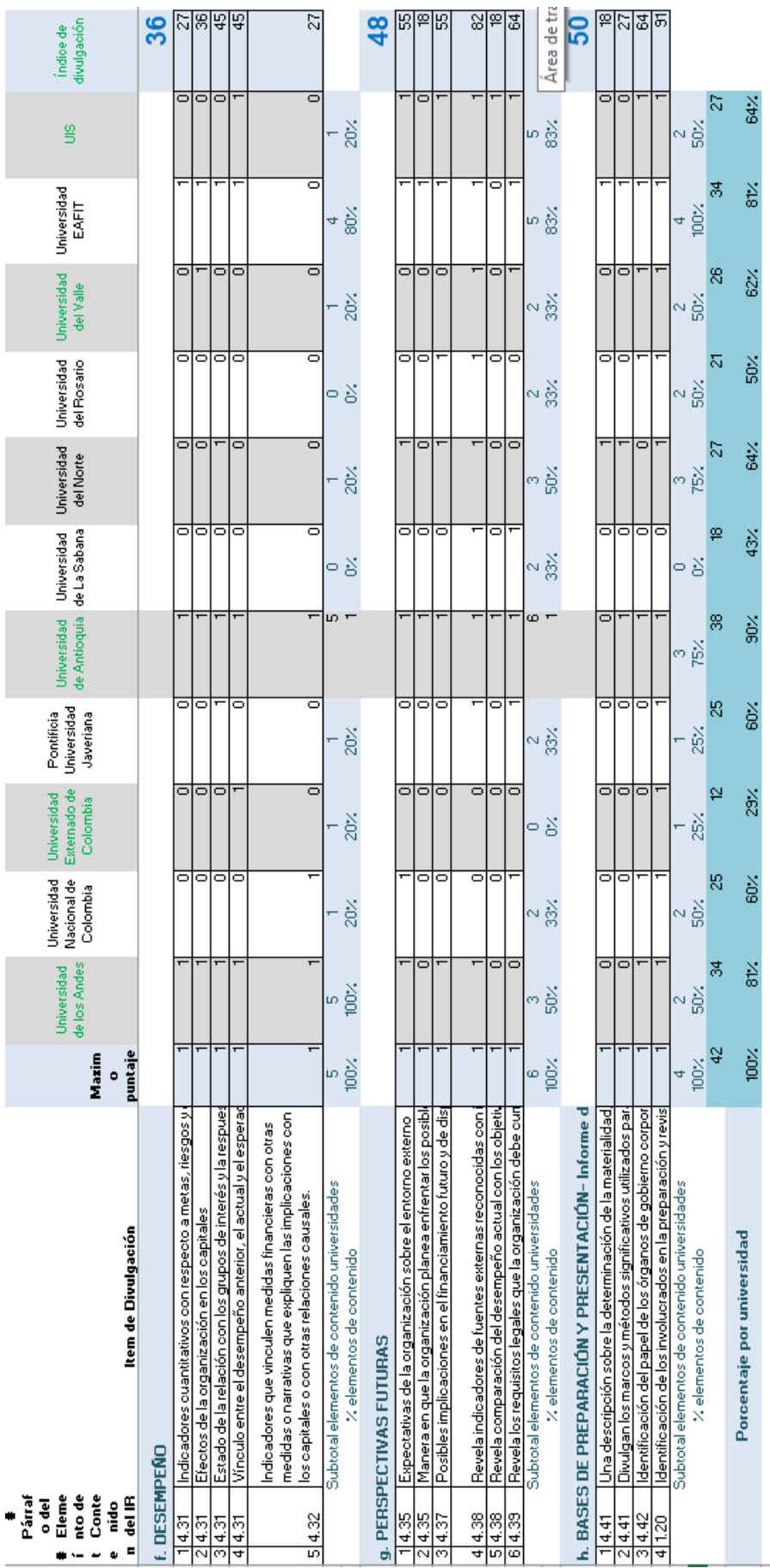

